\title{
Efficient Quality of Service Aware Routing In WSN Using Multi Channel Communication
}

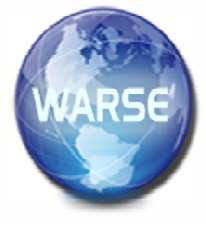

\author{
S.Arockia Babi Reebha ${ }^{1}$,Dr.T.Suresh ${ }^{2}$,Dr.D.Saravanan ${ }^{3}$ \\ ${ }^{1}$ Research Scholar,Assistant Professor,Department of Computer Science \& Engineering, \\ Pavendar Bharathidasan College of Engg.\&Tech, Trichy.sabrphdcse@ gmail.com \\ ${ }^{2}$ Associate Professor, Department of Computer Science \& Engineering, FEAT, \\ Annamalai University, Chidambaram.sureshaucse@gmail.com \\ ${ }^{3}$ Professor, Department of Computer Science \& Engineering, \\ Pavendar Bharathidasan College of Engg.\& Tech, Trichy.dsarav23@ gmail.com
}

\begin{abstract}
This paper focuses on ensuring the quality of service within a Wireless Sensor Network using multi-channel communication and considering the channel interference instead of a single channel communication. A interference aware and probabilistic approach is adopted in this paper to improve the overall efficiency and performance of the network. The topology control and QoS provisioning in IEEE 802.11 based network with dynamic traffic load is studied under a simulated environment. Based on the available transmission links between nodes established by the channel assignment, the set of next highly probable nodes for transmission can be detected. This paper follows the probability theory and extends the channel interference model to give a routing mechanism which implemented using a cross-layer (information from MAC layer sent to routing layer for routing decision) method. The simulation results proved the efficiency of the proposed approach in terms of better packet delivery ratio, throughput, jitter and end to end delay under various network traffic scenarios.
\end{abstract}

Key words: multi-channel communication, probabilistic approach, channel interference, cross-layer

\section{INTRODUCTION}

A WSN - Wireless Sensor Network consists of multiple tiny sensor devices scattered at multiple geographic locations which has limited energy resource (most probably they are battery operated). Each sensor device is equipped with a processor, storage unit and a wireless communication device (transceiver). These devices have the ability to observe the atmosphere or the surrounding environment. The sensed data can be processed and communicated to a destination node referred as the base station which receives and analyzes the data collected from multiple sensor devices. The overall lifetime of a sensor device depends on the remaining energy in the battery source. The radio frequency data transmission consumes most of the energy available in the device[9]. Energy conservation is a main objective in many of the routing protocols design and the main aim in any network is to maintain high Quality of Service (QoS) [7]. Many MAC protocols are designed to listen to the channel at a frequent interval instead of continuously listening the channel's availability for communication [8].
QoS can be defined as a set of requirements in the service to be provisioned in the network during data transmission. QoS can be considered as a measurable level of service provided to the users of the network and it can be characterized by the probability of packet loss, bandwidth availability, and end to end delay in packet transmission. In conventional wired networks the data transmission between the devices are end-to-end in nature without having knowledge on the type and characteristics of the transmitted data. Therefore maintaining end-to-end delay, throughput, and packet loss to an acceptable range will ensure the quality of service. But the same is not applicable in the case of WSN as the sensor device does not communicate in end-to-end fashion rather they transmit the sensed data to the neighboring device and in turn the neighbor nodes forward the data packets towards the destination (sink or base station). The major phenomena to be considered apart from routing the data packet is the energy cost for transmission. The various QoS parameters including coverage and network life time depend on the energy spent during the transmission. The coverage problem may rise when the sensors deployed at different geographic locations couldn't observe the atmosphere and communicate the information to the destination. This can be due to noisy wireless communication channel, or improper management of the network components [10]. The energy cost for transmission depends on the process of finding an optimal route to the destination from the source.

WSN is a scalable network and when the number of sensor devices increases beyond a threshold level then the QoS tend to deteriorate. For this reason designing a optimal routing algorithm and appropriate technique for maintaining QoS in the network is desirable. When provisioning QoS for a WSN network the following factors are to be considered

1. WSN applications are not end-to-end

2. Bandwidth is not a major concern for a single sensor device. Because of the burst nature of the sensor traffic, bandwidth can be an essential consideration for a sensor group.

3. Since multiple sensor devices observe the same environment there is a higher probability of redundancy in the data and hence the packet loss of 
a particular nodes data can be tolerated up to a certain extent.

4. WSN applications are mission critical which shows their importance [1].

QoS provisioning must be scalable and must be able to support a large number of sensor devices, the QoS should not get degraded when the number of sensor increases. The unique characteristics of the WSN poses certain challenges in QoS provisioning when compared to challenges when compared to generic network. WSN applications are growing in large scale and it has become a hot topic for research. QoS of WSN can be characterized by the following factors including reliability, timeliness, availability, and security. The quality of service provided by the network can be quantified by the following parameters such as throughput, end-to-end delay, packet delivery ratio, and jitter. The QoS provisioning mechanism adopted in the network must ensure the implementation of certain strategies to prioritize flow of data packets to the destination [11]. The list of strategies that can be adopted in each of the WSN stack of protocol for QoS provisioning is shown in Table. 1.

Table 1. Summary of strategies for QoS enhancements in WSN

\begin{tabular}{|c|c|c|}
\hline Layer & QoS Strategy & Remarks \\
\hline Application & Compression & $\begin{array}{l}\text { Latency, Available } \\
\text { bandwidth }\end{array}$ \\
\hline \multirow[t]{2}{*}{ Layer } & Adjust sensing rate & $\begin{array}{l}\text { Network Lifetime, } \\
\text { QoS }\end{array}$ \\
\hline & $\begin{array}{l}\text { Data reduction } \\
\text { techniques }\end{array}$ & $\begin{array}{l}\text { Latency, Available } \\
\text { Bandwidth }\end{array}$ \\
\hline \multirow[t]{2}{*}{$\begin{array}{l}\text { Network } \\
\text { Layer }\end{array}$} & Routing protocols & $\begin{array}{l}\text { Reliability, } \\
\text { Latency, N/W } \\
\text { lifetime }\end{array}$ \\
\hline & Packets priority & $\begin{array}{l}\text { Reliability, } \\
\text { Latency, N/W } \\
\text { lifetime }\end{array}$ \\
\hline \multirow{3}{*}{$\begin{array}{l}\text { Physical } \\
\text { Layer }\end{array}$} & Energy Aware MAC & N/W lifetime \\
\hline & $\begin{array}{l}\text { Selection of low } \\
\text { interference channels }\end{array}$ & $\begin{array}{l}\text { Reliability, N/W } \\
\text { lifetime }\end{array}$ \\
\hline & $\begin{array}{l}\text { MAC that avoid } \\
\text { collisions }\end{array}$ & $\begin{array}{l}\text { Reliability, N/W } \\
\text { lifetime }\end{array}$ \\
\hline \multirow[t]{3}{*}{ Link Layer } & Channel Surfing & $\begin{array}{l}\text { Reliability, N/W } \\
\text { lifetime }\end{array}$ \\
\hline & $\begin{array}{l}\text { Modifying signal } \\
\text { power }\end{array}$ & N/W lifetime \\
\hline & $\begin{array}{l}\text { Using low } \\
\text { interference channel }\end{array}$ & $\begin{array}{l}\text { Reliability, N/W } \\
\text { lifetime }\end{array}$ \\
\hline
\end{tabular}

In general the throughput in a wireless network can be increased by following a multi-channel communications when compared to a single channel. The multi-channel communication mode helps to reduce the impact of channel interference. When the sensor devices are equipped with a multi-channel communicable transceiver then each sense the availability and send their data through free channel. Two nodes can communicate when they are using the same channel for communication. The increased throughput is due to the possibility of provisioning permission to parallel transmissions among the neighboring nodes. Whereas in case of a single channel network simultaneous transmissions are not allowed between two neighboring nodes as the channel is shared between the nodes. When multi-channel wireless communication approach is used the problem of collision can be avoided as long as the nodes transmit in different channels. QoS aware routing of data packets in multi-hop wireless channel poses many challenges due to the interference. Channel interference is a major concern even in case of multi-channel wireless network when two nodes attempts to transmit in the same channel [12].

An another important challenge to be addressed in case of wireless networks is to reduce the overhead caused due to transmission of small data packets including the TCP ACKs in case of large scale IEEE 802.11 wireless networks. A suboptimal solution to this issue can be provided by adopting a frame level or packet level aggregation of data packets. Packet aggregation can be implemented at the IP layer without any specific hardware requirements which can be integrated with any wireless networks easily. In an end-toend aggregation category the destination node is capable of de-aggregating the packets. But in contrast the gateway node can be provisioned with methods to de-aggregate the data packets before forwarding it to the destination network. Endto-end aggregation helps to improve the network throughput when constant bit rate is followed.

In a multi-hop network each node attempts to evenly distribute the traffic load to different neighboring nodes thus utilizing the spatial and frequency diversity. In contrast aggregation attempts to combine multiple packets and communicates to one of the neighboring node. Packet reordering has to be executed at the destination node in case of multi-path routing. One of the easiest solutions for packet reordering is to use buffers at the destination node for reordering the packets.

This research paper aims at improving the QoSin a Wireless multi-hop network by adopting a multi-channel communication and following a multi-path routing along with packet aggregation. The objective is to balance the load with in the network by distributing the traffic to multiple hops and reduce the probability of bottleneck nodes. The adopted two approaches have conflicting objectives and may have negative impact on the performance of the network. The algorithm works on the local node and do not add any overhead as no need of transferring extra information between the nodes. End-to-End packet delay may increase as there is a need for packet re-ordering at the receiving node.

\section{RELATED WORK}

Few of the existing researches related QoS provisioning for WSN focus enhancing the life time of the network by energy harvesting from the network environment. This 
facilitates the network nodes to perform more data intensive communication tasks [3-5]. In [14] a multichannel multi-hop communication protocol based on TDMA approach suitable long range transmission is proposed. The throughput in the network is affected by the interference in the channel communication and it is obvious that energy harvesting procedures introduce channel interference problem. In [6] the scheduling algorithm for the data routing and charging tasks is proposed to improve the lifetime of the network nodes and prevent data loss due to channel interference caused by the charging activity.

The available sensor devices in the market have an electronic radio chip (CC2420 / CC2520) which enables the multichannel communication in a WSN node. Multichannel communication ensures high throughput as it supports parallel transmission of data packets through multiple channels. For enhancing the performance of the WSN many of the literatures attempts to lower the issues caused by interference, jamming, and congestion in the network. Multichannel routing protocols including MMSN [15], TMMAC [16] and MCMAC [17] show better performance when compared to single channel protocols [18].

\section{MULTI-CHANNEL BASED PROBABILISTIC ROUTING}

This section presents the polynomial time optimal routing approach followed in this paper which solves the high bandwidth consumption in other routing approaches. Packet flow based route is estimated which cannot be a single path routing mechanism as it not possible to estimate route in polynomial time subject to both inter and intra flow contention. There may be a number of single paths exists between the source node and destination node. The routing problem can be resolved using a probabilistic approach [8]. Let the network topology is represented as $G_{A}=\left(V, E_{A}\right)$ where $\mathrm{A}$ is the wireless channel used for communication. For a new packet delivery request $\rho$ from a sender node $s$, and destination node $t$, and required bandwidth $B$. The aim is to find a route that can be represented by the digraph $\left(G_{A}^{\prime}=\right.$ $\left(V_{A}^{\prime}, E_{A}^{\prime}\right)$ ). The multi access at the MAC layer of each node is handled by the IEEE 802.11 Distributed Coordination Function (DCF). The network has a total of $C$ wireless channels operating at different frequencies and each node is equipped with $Q$ network interface cards where $Q \leq C$. Each node is associated with a fixed transmission power and interference range $R>r$. Each NIC is tuned to communicate in a separate channel to fully utilize the available resources. As described earlier the network can be represented using a directed graph with set of nodes represented as $V$ and set of edges represented as $E$. The wireless link between any two node can be represented by the edge $(u, v)$ in $G$. A channel assignment strategy is followed to assign wireless channel for communication to each node which is denoted as $A(v)$ from the set of available $Q$ wireless channel in the network. The channel assignment defines the topology that is denoted as $G_{A}=\left(V, E_{A}\right)$. An edge $e=$ $(u, v ; k)$ on the wireless channel $\lambda(e)=k$ connecting two nodes $u$ and $v$ can exist only if the distance between the nodes $d(u, v)<\operatorname{rand} \lambda(e) \in A(u) \cap A(v)$. Two nodes of the network may share two or more common channels between them and then $G_{A}$ will include multiple edges from one node to another node.

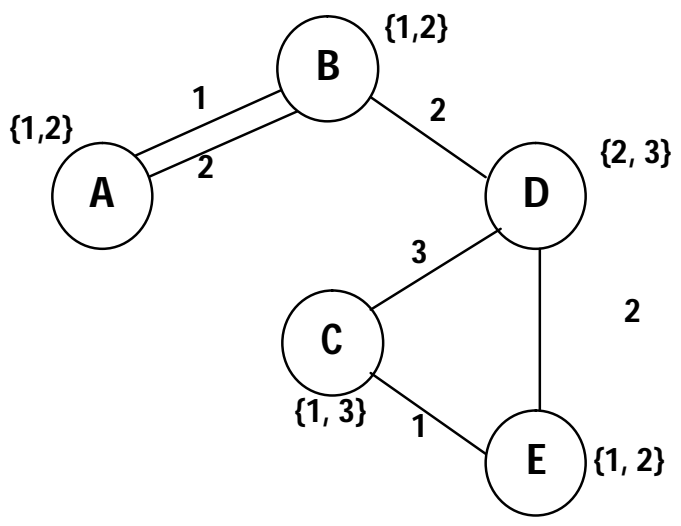

Figure 1. Topology of the Network after Channel Assignment

Figure 1 shows the schematic view of the network topology after channel assignment. In the network only halfduplex communication are allowed to reduce the selfinterference. So that only one NIC out of multiple NIC carried by a node can be used at a time for transmission/ reception. With this topology it is obvious that for any pair of nodes denoted as $u$, and $v$ if $v \in D_{u}$ then $u \in D_{v}$. Presuming that the nodes $u, v, x$,yof the wireless network obeys the following constraint $d(u, v) \leq r$ and $d(x, y) \leq r$ and there exist a common channel $k \in A(u) \cap A(v) \cap A(x) \cap$ $A(y)$. The links $e=(u, v ; k)$ and $e^{\prime}=(x, y ; k)$ share the common channel $k$ and will suffer from collision. Hence it becomes essential to assign channels that define a topology with low interference.

A solution is to identify the next hop node based on the probability of the interference and an interference metric that helps to ensure connectivity among the nodes of the network. The successful transmission of data packets depends on the SINR value estimated at the destination node which is defined as follows

$$
\operatorname{SINR} R_{j}=\frac{P_{i}}{E+\sum_{k \in T_{i}} P_{k}}
$$

Where $P_{i}$ and $P_{k}$ represent the signal power of node $\mathrm{j}$ received from nodes $i$ and $k ; T_{i}$ denotes the set of nodes that attempts to transmit simultaneously with node $j$. For successful transmission of data packets from any node to node $j$, then the SINR at the node $j$ must be less than $\beta$ which is considered as the minimum SINR value that the receiver node should have. The signal receiving power estimated at node $\mathrm{j}$ while receiving a data packet sent from node $\mathrm{i}$ can be defined as $P_{i}=\frac{z P}{\left(d_{i j}\right)^{\lambda}}$ where $\lambda$ represents the signal attenuation factor $(2 \leq \lambda \leq 6), d_{i j}$ is the hop distance between the sender and receiving node. As defined already the transmission range is fixed as Rand the interfering neighboring nodes for a receiver lie within a circle of radius $\mathrm{d}_{\mathrm{L}}$ thend $d_{i j} \leq \frac{d_{L}}{\sqrt[\lambda]{t \beta}}$

With this background the probability that a receiving node will successfully receive a data packet can be define 
mathematically as $\quad=\operatorname{Pr}\left(\operatorname{SINR}_{j}>\beta\right)=(t \beta)^{\frac{-2}{\lambda}\left(\frac{d_{L}}{R}\right)^{2}}$ $\eta=(t \beta)^{\frac{-2}{\lambda}\left(\frac{d_{U}}{R}\right)^{2}}$ is the probability a receiving node successfully receives the packets when interfering nodes lie at a farthest circle of node $j$.

The Network Allocation Vector (NAV) estimated at the MAC layer can be used to approximate the channel occupancy period and PNAV can be defined as the sum of the NAV/ unit time.

$$
P N A V=\frac{\sum_{u \in \Delta t} N A V_{u}}{\Delta t}
$$

The probability of transmitting a data packet at node $i$ can be estimated as follows

$$
\alpha=1-\sqrt[n]{1-\frac{\sum_{u \in \Delta t} N A V_{u}}{\Delta t}}
$$

Here NAV can be read from the MAC layer and sent to the routing layer and the maximum communication range $R_{\text {max }}$ can be defined as

$$
R_{\max }=\sqrt[\lambda]{\frac{Z P}{E \beta}}
$$

Considering the interference at the sending and receiving node the routing metric can be defined as

$$
\xi=\sqrt[2]{\alpha \cdot \frac{\tau+\eta}{2}} \sqrt{\frac{R_{\max }}{R_{\max }+d_{j \sin k}}}
$$

where $d_{j \operatorname{sink}}$ denotes the distance between the node $\mathrm{j}$ (neighboring node of sender) and the destination node. The routing decision is taken by estimating the probability of transmitting a data packet successfully from the sender node to a destination node. The sender and every intermediate node on the routing path select the next hop node based on the estimated probability. The neighboring node with the highest probability is selected as the next hop node for transmission.

\section{SIMULATION AND RESULTS}

For evaluating the performance of the probabilistic routing approach the simulations are done in network simulator. The density of nodes in the network is varied between 300 and 600 with in a network region of $300 \times 300$ $\mathrm{m}$. The maximum communication range of node is fixed as $50 \mathrm{~m}$. Multiple nodes are allowed to transmit at the same instance as each node is configured with multi-channel wireless radio. During the simulation the sending rate is varied and the performance of the proposed model is evaluated. The total duration of the simulation is conducted for a period of 500 seconds. The simulation includes an analysis of multiple sources and a single sink. The performance of the network is analyzed using the following metrics including jitter, average delay, and average transmission route length under various network conditions. Figure 2 shows the performance of the probabilistic routing is compared with the performance of the multi-path AODV.

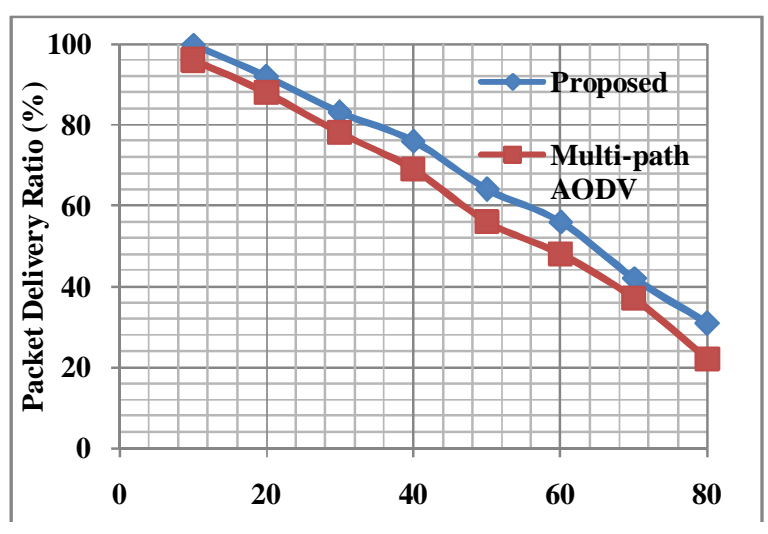

Figure 2. Comparison of Packet Delivery Ratio

The variation in the packet delivery ratio when the transmission rate is varied during the simulation of the network is presented. The higher packet delivery ratio is achieved in case of proposed probabilistic based routing approach as it considers the interference in the route discovery process.

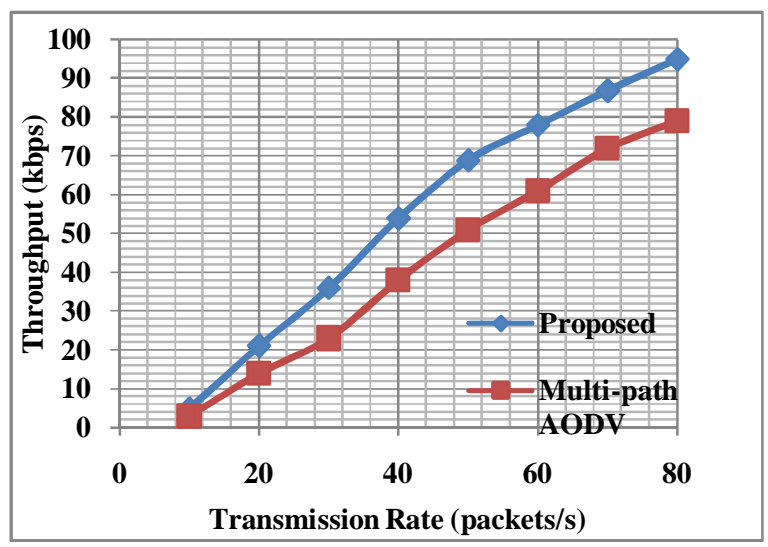

Figure 3. Throughput Analysis

Figure 3 shows Throughput analysis is presented. The throughput is plotted against the transmission rate in the $\mathrm{x}$ axis. The proposed approach is capable of sending more packets in a period of time as it considers the interference during the routing process.

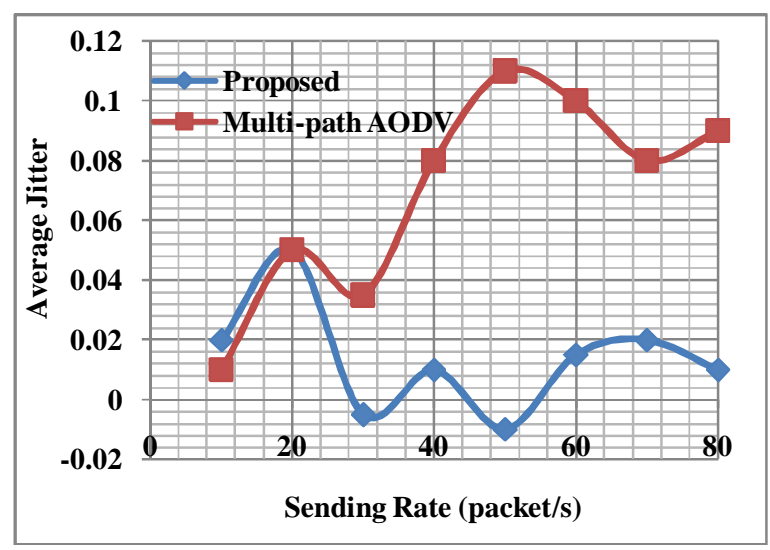

Figure 4. Plot of Average Jitter 
Figure 4 shows the average jitter of the proposed approach is smaller when compared to that of the multipath distance vector routing.

\section{CONCLUSION}

A multichannel interference aware topology control and QoS enhanced routing in IEEE 802.11 based multichannel WSN is studied. A probability based routing mechanism is derived which enabled the sending network nodes to discover the routing path one hop at a time. The proposed approach is implemented using cross layer design as the network allocation vector read from the MAC layer is communicated to the routing layer for estimating the sending probability. This approach considers both the sender and receiver interference levels and estimates a routing metric which is used deciding the next hop node in the routing path. The simulation results exhibited the superior performance of the proposed approach when compared to a conventional multipath routing protocol.

\section{REFERENCES}

[1] S. Kumar, Z. Feng, F. Hu, Y. Xiao, "E2SRT: enhanced event-to-sink reliable transport for wireless sensor networks," Wireless Communications and Mobile Computing, Volume 9, Issue 10, pages 1301-1311, October 2009.

[2] Lu, J. and Wang, X., 2012. Interference-aware probabilistic routing for wireless sensor networks. Tsinghua Science and Technology, 17(5), pp.575-585.

[3] Tang, Q.; Yang, K.; Wang, J.; Luo, Y.; Li, K.; Yu, F. Wireless Sensor Network MCDS Construction Algorithms with Energy Consideration for Extreme Environments Healthcare. IEEE Access 2019, 7, 3313033144.

[4] Cao, D.; Zheng, B.; Ji, B.; Lei, Z.; Feng, C. A robust distance-based relay selection for message dissemination in vehicular network. Wirel. Netw. 2018, $1-17$.

[5] He, S.; Zhang, D.; Xie, K.; Qiao, H.; Zhang, J. Distributed low-complexity channel assignment for opportunistic routing. China Commun. 2012, 9, 9-22.

[6] Tan, Q., Qu, Y., Xu, K., Wang, H., Wang, D. and Shen, M., 2019. Interference-aware lifetime maximization with joint routing and charging in wireless sensor networks. CCF Transactions on Networking, 2(3), pp.188-206.

[7] Akyildiz, I. F., Su, W., Sankarasubramaniam, Y., and Cayirci, E. Wireless sensor networks: a survey. IEEE Communications Magazine 40, 8 (August 2002), 102114.

[8] Willig, A., and Karl, H. Data transport reliability in wireless sensor networks - a survey of issues and solutions. In Praxis der Informationsverarbeitung und Kommunikation (April 2005), vol. 28, pp. 86-92.

[9] Estrin, D., Girod, L., Pottie, G., and Srivastava, M. Instrumenting the world with wireless sensor networks. In International Conference on Acoustics, Speech, and Signal Processing (ICASSP 2001), Salt Lake City, Utah, May 2001. (2001).
[10] Chen, D., and Varshney, P. K. QoS support in wireless sensor networks: A survey. In Proc. of the 2004 International Conference on Wireless Networks (ICWN 2004) (Las Vegas, Nevada, USA, June 2004), vol. 1, pp. 227-233.

[11] D Petrović, R Shah, K Ramchandran and J Rabaey, "Data Funneling: Routing with Aggregation and Compression for Wireless Sensor Networks" In Proceedings of First IEEE International Workshop on Sensor Network Protocols and Applications, Page(s): 156- 162, May 2003.

[12] Tang, Jian, GuoliangXue, and Weiyi Zhang. "Interference-aware topology control and QoS routing in multi-channel wireless mesh networks." In Proceedings of the 6th ACM international symposium on Mobile ad hoc networking and computing, pp. 68-77. 2005.

[13] Tang, J., Xue, G., \& Zhang, W. (2005, May). Interference-aware topology control and QoS routing in multi-channel wireless mesh networks. In Proceedings of the 6th ACM international symposium on Mobile ad hoc networking and computing (pp. 68-77).

[14] Toldov, Viktor, Laurent Clavier, and Nathalie Mitton. "Multi-channel Distributed MAC protocol for WSNbased wildlife monitoring." In 2018 14th International Conference on Wireless and Mobile Computing, Networking and Communications (WiMob), pp. 1-8. IEEE, 2018.

[15] G. Zhou, C. Huang, T. Yan, T. He, J. A. Stankovic, and T. F. Abdelzaher, "Mmsn: Multi-frequency media access control for wireless sensor networks," in 25th IEEE International Conference on Computer Communications, ser. INFOCOM '06, vol. 6, April 2006, pp. 1-13.

[16] J. Zhang, G. Zhou, C. Huang, S. H. Son, and J. A. Stankovic, "Tmmac: An energy efficient multi-channel mac protocol for ad hoc networks," in IEEE International Conference on Communications, ser. ICC '07, June 2007, pp. 3554-3561.

[17] X. Chen, P. Han, Q.-S. He, S.-L. Tu, and Z.-L. Chen, "A multichannel mac protocol for wireless sensor networks," in The Sixth IEEE International Conference on Computer and Information Technology, ser. CIT '06, September 2006, pp. 224-224.

[18] Y. Wu, J. Stankovic, T. He, and S. Lin, "Realistic and efficient multichannel communications in wireless sensor networks," in The 27th IEEE Conference on Computer Communications, ser. INFOCOM '08, April 2008. 